\title{
Wissenschaftliche Originalarbeiten in der SÄZ
}

In dieser Ausgabe der Schweizerischen Ärztezeitung (SÄZ) finden Sie eine wissenschaftliche Originalarbeit [1]. Texte dieser Art werden gemäss Autorenrichtlinien in der SÄZ nur ausnahmsweise publiziert.

Es gibt verschiedene Gründe, warum die SÄZRedaktion bei der Annahme von wissenschaftlichen Arbeiten eine restriktive Politik verfolgt. Gemäss Redaktionsstatut hat die SÄZ zunächst die Funktion des offiziellen Organs der FMH, alle Ärzte und medizinisch
Ressource für die Erhebung von sicherheitsrelevanten Ereignissen darstellen, und dass dies bei Risikomanagern im Spital auf grosses Interesse stösst. Folgende Argumente haben die Redaktion überzeugt, diesen Artikel in der SÄZ zu veröffentlichen: Das Projekt wurde von der Stiftung für Patientensicherheit in Auftrag gegeben; es erscheint wichtig, dass die Ärzteschaft zur Kenntnis nimmt, wofür Bund, mehrere Kantone und die FMH sich gemeinsam einsetzen. Die Autoren sind

\section{Ist das Dilemma der Redaktion für Sie nachvollziehbar? Wir sind auf Ihre Meinung gespannt!}

1 Frank O, Hochreutener M, Schwappach D. Patientensicherheits-Benchmarking in Schweizer Spitälern. Schweiz Ärztezeitung. 2010;91(9):363-6.

2 Harringer O, Hoby G Patientenverfügung in der ärztlichen Praxis. Schweiz Ärztezeitung. 2009;90(8):303-4 und PrimaryCare 2009;9(3):56-8.

* Prof. Dr. med. Hans Stalder Facharzt für Innere Medizin FMH und Mitglied der Redaktion, ist ehemalige Chefarzt der medizinischen Poliklinik und des Département de Médecine communautaire am Universitätsspital Genf.

** Dr. med. et lic. phil. Bruno Kesseli, SÄZ-Chefredaktor, ist Facharzt für Allgemeinmedizin FMH sowie Literatur- und Publizistikwissenschaftler. Vor seinem Stellenantritt war er neben seiner ärztlichen Tätigkeit unter anderem als Medizin- und Wissenschaftsjournalist für

Printmedien tätig.

hans.stalder@saez.ch

bkesseli@emh.ch interessierten Personen über Berufsprobleme und Standespolitik zu informieren. Sie dient zudem als freie Diskussionsplattform zu Fragen des ärztlichen Berufs und des Gesundheitswesens - dies in einem umfassenden Sinn, unter Einschluss ethischer, juristischer, ökonomischer, historischer und politischer Dimensionen. Auch die Pflege kultureller Themen aus dem weiteren Umfeld des Gesundheitswesens ist vorgesehen.

Was die Art der Vermittlung von wissenschaftlichen Inhalten betrifft, orientiert sich die SÄZ konzeptuell viel mehr an anspruchsvollen Publikumszeitschriften als an wissenschaftlichen Journals. Ihre redaktionellen Strukturen sind nicht auf kompetente Beurteilung wissenschaftlicher (Original-)Arbeiten ausgerichtet. Innerhalb der Editiones Medicorum Helveticorum ist für wissenschaftliche Publikationen das Swiss Medical Weekly (SMW) zuständig. Für die SÄZ steht die Konzentration auf ihre Kernaufgaben klar im Vordergrund - sie sollte nicht zum MWJ (Medical Waste Journal) werden, das Autoren «dank» eines fehlenden Reviewer-Netzwerks die Möglichkeit bietet, von anderen Journals abgelehnte Artikel zu publizieren.

Warum trotzdem Ausnahmen? Es gibt immer wieder Artikel, die für ein sehr breites Publikum interessant sind und auch Leser erreichen sollten, die kaum je wissenschaftliche Journals in die Hand nehmen. Ebenso rechtfertigt sich gelegentlich die Publikation wichtiger Aussagen wissenschaftlicher Art, die von Praktikern gefunden wurden, die davor zurückschrecken, sich den geschriebenen und ungeschriebenen Gepflogenheiten wissenschaftlicher Journals zu unterziehen oder ihre Resultate ins Englische zu übersetzen - das SMW nimmt ja (leider?) keine Artikel in unseren Landessprachen an. Solche Artikel können sogar einen Forschungspreis erhalten [2].

Die Arbeit von O. Frank, M. Hochreutener und D. Schwappach [1] zeigt, dass Patienten eine wichtige international anerkannt und bieten somit eine gewisse Gewähr für die wissenschaftliche Qualität der Arbeit, zumal diese in einer anderen Fassung einem Peer-reviewed Journal unterbreitet worden ist. Dazu kommt, dass die Studie in der Schweiz durchgeführt worden ist und in der Schweiz einem breiten Kreis bekannt gemacht werden sollte.

Und trotzdem bleiben gewisse Zweifel: Ist die Arbeit wirklich so originell, wie sie in der ersten Wahrnehmung scheint, oder repetiert sie nur, was andere schon längst gefunden haben? Wurde der Fragebogen wirklich korrekt validiert? Ist die Rücklaufquote von $55 \%$ genügend, oder besteht ein Non-response bias? Braucht es eine Patientenbefragung, um zu zeigen, dass Phlebitiden, nosokomiale Infektionen und mangelnde Händehygiene relevant sind für die Patientensicherheit, oder war das nicht schon längst bekannt? Hat die Arbeit wirklich die «Risikomanager»- sind es Ärzte oder Verwalter? - dazu gebracht, messbare Verbesserungen im Spital durchzusetzen, die nicht schon auf andere Weise angeregt worden waren? Und schliesslich: Besteht ein ethisches Problem, wenn Patienten im Sinn von «Kunden» benützt werden, um Fehler zu orten, auch wenn dies nachträglich geschah? Wird dadurch nicht das Vertrauen gegenüber den Pflegenden, das ja allgemein für die Heilung als wichtig angesehen wird, beeinträchtigt? Übrigens: Wurde das Projekt einer Ethikkommission unterbreitet?

Es wäre interessant, zu erfahren, wie Sie, liebe Leserinnen und Leser, zu diesen Fragen stehen und ob Sie der Ansicht sind, dass die SÄZ weiterhin (ausnahmsweise) wissenschaftliche Artikel veröffentlichen sollte? Ist das Dilemma der Redaktion für Sie nachvollziehbar? Wir sind auf Ihre Meinung gespannt!

Hans Stalder* und Bruno Kesseli** 